\title{
8
}

\section{Humanities and Public Policy}

This chapter deals with the interaction of the humanities with the political system. How do humanities representatives argue the case for the humanities, and what do political systems expect of the humanities? We shall examine various policy documents, such as national reports and international research programmes designed by non-academic bodies. The aim is to identify how humanities' interests are being voiced and promoted and what the regional characteristics of the public role of the humanities are. We concentrate on US and EU cases specifically but will make some observations on other regions for contrast. The information for US, EU, China and India is drawn from our own desk research, while information for other regions is based on country reports for the EU METRIS project.

Political processes involve many actors and motives. We do not pretend to present in-depth analyses but simply identify some of the main advocates for humanities funding, and evaluate the political take up as expressed by budgets (given that funding levels may be a better proxy of goodwill than stated intentions). This chapter pays special attention to humanities policies in Europe as the EU and a few national governments combined provide by far the largest public funds for humanities research globally. As most of these funds are open to non-EU citizens they attract worldwide attention. This chapter raises questions of the quid pro quo when the humanities engage with societal concerns. The potential benefits of increased funding may come with questions about the independence of the research.

\section{The United States}

Generally, American universities are funded by a mix of student fees, endowments and state funding. In addition, researchers rely on public 
and private foundations for competitive grants. Public funding - federal and state - is generally much lower than in most other countries in the world, and humanities public policy is therefore also quite different from most other countries. We limit ourselves to a discussion of the role of federal funding as individual states may differ very much in their support of universities. It should be noted that while most states have shrunk their funding of the university sector considerably, states like California, New York and Michigan still make substantial funds available to the sector and indirectly to humanities research.

The main US public foundations for research, the National Science Foundation (NSF) and the National Institute for Health (NIH), by and large do not fund humanities research. It may throw some light on the political expectation of the usefulness of the humanities to consider the vote by Congress in March 2013 to limit funding for political science in the budget of the National Science Foundation (NSF). In the language of Senator Coburn who proposed the amendment, this was to 'prevent wasting federal resources on political science projects, unless the NSF Director certifies projects are vital to national security or the economic interests of the country'. ${ }^{1}$ The Senate carried the amendment without opposition. We have found no recent direct policy statement on the role of the humanities in and for society. Considering the enormous soft power of American culture this may be seen as an anomaly but it probably simply reflects the fact that private enterprises in the global market have promoted American culture unchallenged since the Second World War. It is also important to note that in the USA elite universities are well-endowed and private foundations play a large role in supporting humanities research. Most public resources are directed to, so-called, STEM research (science, technology, engineering, and mathematics), whereas very little money is funneled to the humanities.

In light of this, it may be understandable that the US National Endowment for the Humanities (NEH) couches its budget request to Congress in very defensive terms: 'While many still think of humanities research as a dusty, unchanging, and solitary endeavor - the lone scholar ardently sifting through archives and libraries - scholarly research in the 21st century is dynamic. It is grounded in traditional scholarly methods and best practices, but informed by international networks of scholars and enhanced by new methods of accessing vital documents.' The NEH avoids talking about the role of the humanities in society except in very broad terms. Its mission is summed up in this way on its website homepage: 'Because democracy demands wisdom, $\mathrm{NEH}$ serves and strengthens our republic by promoting excellence in the 
humanities and conveying the lessons of history to all Americans.' The NEH Strategic Plan 2013-17 identifies its role as to serve and strengthen 'our nation by supporting high quality projects and programmes in the humanities and by making the humanities available to all Americans.' The NEH budget request argues 'Many NEH-supported projects have direct relevance to current events', and in support of this claim lists books on the Taliban, the Darfur crisis, financial history and global power balance. It further argues that there is a strong public interest in American culture and the Civil War, serviced by NEH-funded books. The books singled out for mention tend to address themes of contemporary topical interest in the American news media.

The NEH's annual budget of around 150 million USD amounts to probably around $0.45 \%$ of the federal research budget. ${ }^{2}$ The budget of the NEH waxes and wanes as congressional majorities change, and the Republican Party has, at times, attempted to abolish the funding altogether, most clearly in $1998 .^{3}$ The NEH budget is divided between eight grant-making offices, only one of which directly funds research with an allocation in 2012 of 14.5 million USD. Most of the rest of NEH funding goes to community college programmes, teacher support, outreach and infrastructural programmes, which may include some research funding. ${ }^{4}$ In total, we would estimate that, at best, some 25 million USD funds direct research. It should be mentioned that $80 \%$ of awardees report that the NEH award enables them to leverage additional support from their employers or another funder.

The NEH primarily funds 'research by individual scholars (fellowships, summer stipends, documenting endangered languages, and awards for faculty); long-term, complex projects carried out by teams of scholars (scholarly editions and translations and collaborative research); and focused, individual projects that draw upon the collections and expertise of leading humanities institutions and overseas research centers (Fellowship Programs at Independent Research Institutions)'. The NEH does have a budget line for collaborative research but the funds are small. The fellowship programmes would mostly be in support of multiple individual scholars. In 2012 the NEH carried out a full-scale evaluation of the long-term outcome of awards made from 2002 through 2004. Over $96 \%$ of the awards resulted in a publication and $70 \%$ in a book. Although the NEH flags its support for new work models the funding seems overwhelmingly to be in support of individual scholars writing a book.

While the NEH is reluctant to engage in direct public advocacy of the usefulness of the humanities to society except in the broad terms 
indicated above, more explicit statements are made in the 2013 report for the American Association for the Advancement of Arts and Science, The Heart of the Matter. The report particularly underlines the value of the humanities for American society and security. The humanities (and social sciences) are said to be a 'source of national memory'. They 'remind us where we have been and help us envision where we are going', ${ }^{5}$ they guide us to respect 'communalities and differences' between people of the world, ${ }^{6}$ and '... they help us understand what it means to be human and connects us with our global community'. ${ }^{7}$ All these specific assets should be employed in order to make competent, self-fulfilling and independent citizens of people, reach out to citizens, integrate with other fields of research and respond to the grand challenges. Humanist scholars are encouraged to respond positively, both to challenges common to humankind in general, such as clean air and water, food, health, energy, universal education, human rights and physical safety, ${ }^{8}$ and to specific national ones, such as US war missions and the preservation of its leadership in the world. ${ }^{9}$ Basically, it is stated, everything 'scholars do to connect with the broader public advances their case for support, and everything they neglect to do weakens that case'.$^{10}$ The report emphasises the importance of the humanities to understand 'foreign histories, social constructs, belief systems, languages and cultures' for providing 'experts in national security, equipped with the cultural understanding, knowledge of social dynamics, and language proficiency to lead our foreign service and military through complex global conflicts'. ${ }^{11}$

In conclusion, the American funding system reflects a perception of the humanities as detached from direct importance to ongoing political concerns. The NEH emphasis on the role of wisdom as the main contribution by humanities to society is interesting. Although some humanities advocates identify an applied use of some humanities knowledge in strengthening homeland security, the underlying impression is one of a disconnection between the humanities and society, agreed by both parties.

\section{China}

In China there seems to be increasing political interest in the humanities. As noted in previous chapters funding is increasing, there is a strong interest in building networks and inviting scholars from abroad, and leading universities are developing humanities programmes fast. We have not conducted an in-depth study of the Chinese policy behind 
these developments but, in addition to growing student interest, there is no doubt that there is increased high-level political interest in the field. We are not aware of any open policy documents but impressions from visits by the authors to the country leave a sense that there are both economic and political values in play. The economic value of the humanities seems to be related to a sense that future growth will depend not only on technological progress and the availability of labour but also on well-educated professionals with sophisticated taste and an ability to overcome cultural and linguistic barriers. In this respect Chinese politicians seem to realise that there is a need to invest in human cultural capital as a means to sustain future growth. A second impetus for increased investment in the humanities is Chinese foreign policy, which includes a focus on soft power. The remarkable establishment of more than a hundred Confucius Institutes in major cities and universities across the globe is an indication of the priority put on developing an understanding of China's role in the world by sustaining cultural interaction. Western intellectuals have criticised the Confucius Institutes as vehicles for an antidemocratic regime and some potential host universities have declined an offer to establish an institute. In this respect, the positive development of the humanities in China comes with very real concerns about issues of free speech and thought.

\section{South Africa and Australia}

In South Africa and Australia the humanities seem to have a recognised societal importance, which is not however fully developed and in recent years may even have diminished. A public policy report on the humanities in South Africa stands out for stressing the importance of the humanities to social cohesion. The report claimed that societal problems of national concern, such as 'violence, corruption, innovation, the gap between rich and poor, the issue of race' all have 'their solutions ... in the Humanities' ${ }^{12}$ It is also stated that the humanities, dealing with 'human communication', tell 'us who we are' and teach us 'to see other ways of seeing', as in the social value of tolerance towards others. ${ }^{13}$ Knowledge about interlocutors from different cultures gained in anthropology, sociology and cultural studies, is put into practical use in diplomacy, journalism, teaching and so on, ${ }^{14}$ proving the social value of the humanities (in this case very broadly defined to include parts of the social sciences). The South African report also pointed out that the humanities build bridges between past and present, particularly 
referring to archaeology research. ${ }^{15}$ In contrast, the economic value of the humanities, or rather policy makers' stress on economic growth, was not only downplayed but thoroughly criticised for being narrowly utilitarian; instead of building community, this goal has atomised society, bringing with it a series of problems - global warming, global poverty, global epidemics - which can no longer be managed but will only be solved through the community-centred perspectives championed by the humanities. ${ }^{16}$ 'Building community', or the promotion of the common good, is seen as the very essence of the mission of the humanities. ${ }^{17}$

The South African report is striking, not only for claiming a societal role for the humanities but also by being critical of the current paucity of society's expectations of the humanities. The report stated that, originally, the humanities were thought of as 'interpreters (facilitating an understanding of social process and social innovation), change agents (facilitating technological change), generators of policy, critics and producers of knowledge, and finally as educators...', but current use has narrowed their role down to being just 'handmaidens of innovation activities initiated in other science domains'. ${ }^{18}$ According to the report, there are no longer any references to the humanities making scientific contributions 'in their own right'. ${ }^{19}$ In turn, this has meant a declining support of the humanities in relation to other fields. The report also observed that the post-1994 period of democratic reconstruction, and reorientation of science policy, saw a progressive narrowing of the role of humanities disciplines, a growing instrumentalisation of the humanities in the service of innovation, and a decline in funding and the support base. The report found that this detrimental development for the humanities in the NSI (National System of Innovation) of South Africa may be explained by a lack of serious intellectual engagement with this conceptualisation by academics and researchers, and a lack of humanities' champions in the Department of Science and Technology and the Department of Education to argue their use. ${ }^{20}$ We cannot assess these assertions but they do seem to be corroborated by government policy. The National Research Plan of 2009 mentions the humanities only once, as a conditional and auxiliary addendum to research on global human-ecological change. ${ }^{21}$

In Australia questions of aboriginal rights and environmental concerns have given prominence to the humanities. In recent years the Australian Academy of the Humanities (AAH) has submitted five to seven documents annually, advising on issues such as national research priorities, research training, cultural policy, research infrastructure, excellence in research and the future of scholarly publishing. 
The AAH is a government-funded independent organisation of some 500 fellows and one of its key roles is to provide 'independent, expert advice to government, industry, the media, cultural organisations and community groups'. The visibility of the humanities does expose it to political counter-attacks, however, and one of the political aims of the new Liberal government, which came to power in 2013, was to abolish federal funding for 'wasteful' and 'ridiculous' humanities research. It publicly highlighted research to be scrapped, such as 'sexuality in Islamic interpretations of reproductive health technologies in Egypt', 'how urban media art can best respond to global climate change' and 'The God of Hegel's Post-Kantian idealism'. ${ }^{22}$ The Australian case highlights that political attention may come at a cost.

\section{India, Japan and Latin America}

In India and Japan the humanities seem to be low on the political agenda. The India Foundation for the Arts (IFA) provided a report mapping the humanities in $2010 .^{23}$ It contrasted the funding position of the social sciences with the humanities and concluded that the humanities in India were not flourishing and were inadequately supported. While the social sciences were better off due to the existence of specialised agencies, corporate interest and project-based funding from the World Bank and other foreign institutions, the humanities relied on government funding by the Indian Council of Historical Research (ICHR) and the Indian Council of Philosophical Research (ICPR). The budget of the ICHR budget was 106 million Rs in 2008-9 and has increased since, while the ICPR reported a declining budget of 63 million Rs in 2009-10. Relative to the size of the academic communities of the country the budgets are very small. According to the IFA report, 'professional philanthropy in the arts and humanities in India is still a nascent area' (p. 27), although clearly growing with the Sir Ratan Tata Trust, which funds some major humanities research centres. The report also stated that funding for literature and languages has 'shrunk considerably' (p. 8). In general, the mapping indicated that the social sciences had taken a privileged place in India, linked to public welfare policies, while the funding for the humanities and their societal role remained limited.

Japan has one of the largest numbers of humanities graduates in the world and new large-scale infrastructures and projects, which specifically address humanities subjects, are underway in geospatial and digital applications. Nevertheless, the humanities do not seem to be called on 
to address the huge demographic, environmental and economic issues that the country has faced in the last two decades. Science and technology studies are viewed as the best bets for the future. ${ }^{24}$

Relative neglect seems also to be the case in Latin America. In Brazil the National Council of Science and Technology seems to have limited interest in drawing on the social sciences, not to speak of the humanities. ${ }^{25}$ The HWR workshop on Latin America did point to questions of social cohesion and religion as areas where the humanities have had a societal impact, not least in Mexico, but overall governments seem little interested in mobilising the humanities for policy advice.

\section{The European Union}

In Europe, on the other hand, the language and reality are very different from the American situation, despite the fact that many scholars have close ties across the Atlantic. While the single states of the US have very limited, or non-existent, budgets for the humanities, most of the national states of Europe have significant public humanities budgets. In terms of soft power Europe is not one entity but many national cultures, each with varying takes on the humanities. National research budgets in north west European countries, such as Germany, France, UK, Benelux and the Nordic countries are substantial and far outweigh the importance of the EU contribution to humanities research in these countries, while EU funding is crucial to many southern and eastern countries. The challenges of a communist past in Eastern Europe and the postcolonial realities of countries like the UK, Spain and Portugal also contribute to European diversity and global reach. There are substantial private foundations for the humanities in some countries, such as the Volkswagen Stiftung in Germany, the Leverhulme Foundation in the UK and the Carlsberg Foundation in Denmark. However, relative to the American tradition of private funding, humanities scholars in Europe must look more to the state for funding.

With few exceptions, European countries (EU and non-EU) have research prioritisation plans that identify policy-relevant research topics. Some of these topics explicitly call on the humanities although funding is typically limited relative to science budgets. A review of these national priorities reveal a few top areas as listed in Table 8.1.

Other main areas include topics like behaviour and cognition, democracy, families and lifestyles, while topics like conflict and peace studies, gender, globalisation, migration and international relations are prioritised by only a couple of countries. 
Table 8.1 European humanities-relevant national research priorities

\begin{tabular}{lc}
\hline Priorities & Number of countries (total 29) \\
\hline Education, lifelong learning & 16 \\
Social cohesion, inequality, poverty & 14 \\
Cultural heritage & 14 \\
Sustainable development & 12 \\
Health & 11 \\
Identity, religion, language, multi- & 10 \\
culturalism & \\
\hline
\end{tabular}

Source: METRIS dashboard, policy priorities. http://www.metrisnet.eu/metris/index.cfm/init/ dashboard.

The European Union of 29 countries in 2014 is increasingly a funder and policy maker for the humanities. In principle, research is funded by the nation states, and European funding is only allowed when a case can be made for European added value of joint funding. In accordance with the European Treaty all funding must contribute to European Union aims, primarily economic growth and European integration. The research budget has increased rapidly as knowledge and innovation has been identified as the main competitive factor in the global market. In financial terms the EU budget allocates more money to the humanities than the US, even though comparisons are difficult. The European Parliament votes on the research budget every seven years. Since 2007 the research programme has included support for free, bottom-up research, as proposed by the researchers themselves, and strategic or policy-oriented top-down programmes.

The European Research Council (ERC), which grants the awards for bottom-up free research, acknowledges the humanities as part of the sciences. Significantly, the programme is open to applicants from any country in the world and $17 \%$ of its budget goes to the humanities and social sciences, or about 325 million euro per year projected for the seven-year period from 2014 under the new Horizon 2020 programme. Recent practice shows that the money is split equally between the two domains, making some 160 million euro per year available for humanities research. Other 'free' money is made available through the Marie Curie programme for researcher mobility, which funds a substantial number of humanities researchers. Based on past shares, the humanities may expect funding of around 36 million euro per year. The humanities share of the top-down funding stream is much more difficult to estimate. The budget of Social Challenge 6, which will include the bulk 
of targeted humanities research, is unlikely to exceed 50 million euro annually. In addition, some humanities researchers will receive funding as partners of multi-disciplinary teams including the natural sciences and engineering. A rough total estimate of EU funding for bottom-up and top-down humanities research is around 250-80 million euro per year. This would indicate that EU expenditure on humanities research is ten times that of the US federal budget, as calculated above. ${ }^{26}$ Still, the humanities share of total EU research funding is only about $2 \%$.

In the EU, the social sciences and humanities are usually treated together as a single SSH field. The inclusion of the humanities is a fairly recent phenomenon as part of a gradual widening of the remit of the EU research budget. It is of interest to study in some detail the development of the humanities policy agenda. In 1994 the European Council decided to launch the Targeted Socio-Economic Research programme to provide evidence-based knowledge for science and technology options, education and social integration. ${ }^{27}$ The launch of the Sixth Framework Programme in 2000 inaugurated broader support for social science research. The inclusion of the social sciences encouraged national research councils, primarily in Denmark, the Netherlands and Ireland, to join forces to raise the voice of the humanities. A conference in 2002, 'Humanities Essential Research for Europe', gathered some sixty representatives of national research councils and academies and agreed a mandate for a European Network of Research Councils for the Humanities (ERCH). The objective of the ERCH was to 'work to strengthen the Humanities in Europe at the political and organisational level' and a declaration identified four action points for the national councils at the European level:

- to stimulate and focus basic research in the humanities, e.g. by doing comparative research

- to develop a European research infrastructure for the humanities

- to increase the role of the humanities in future integrated projects of the European Framework Programme

- to define the role of the humanities in the European Research Area and in particular to identify and build structures to achieve this aim. $^{28}$

The Odense Declaration identified an agenda for the humanities for the next decade. As a direct result, the EU Commission for Research invited the ERCH to apply for matching funds to set up a pilot scheme for humanities funding at the European scale, primarily addressing the first and last of the action points above. In 2005, on receipt of EU 
funding, the ERCH morphed into HERA (Humanities in the European Research Area), a partnership that now consists of 21 national funding bodies in Europe. To date HERA has launched three thematic calls, mostly funded by the partners but also by a third of the total funding costs paid by the European commission as a part of its ERA-NET initiative to develop the European Research Area as an entity. Two of them were launched simultaneously, one on 'Cultural Dynamics - Inheritance and Identity', the other on 'Humanities as a Source of Creativity and Innovation'.$^{29}$ The first was an invitation to humanist scholars across Europe to study 'the way in which cultural exchanges and dynamics cross between social strata, between countries, and between media'. Three topics were outlined, the first about collective 'identities before and after the nation-state', the second on culture 'as self-reflection' and the third cultural "practices between "high" and "low", local and global, performance and ownership'. The intention of the second call was to generate new knowledge and develop new perspectives on creativity and innovation research. In 2012 a new programme was launched, rather similar to the first one, called 'Cultural Encounters', with a focus on peaceful and conflict-ridden encounters between people from different cultures. ${ }^{30}$

The second action point, infrastructure for the humanities, informed humanities action in ESFRI, the European Strategy Forum on Research Infrastructures. This body was set up by the Commission and the Member States in 2002 to develop a joint strategy for future investments. While ESFRI was not intended to include the humanities, active lobbying secured the inclusion of two important facilities: the Common Language Resources and Technology Infrastructure (CLARIN), with an estimated operational cost of 7.6 million euro per year; and the Digital Research Infrastructure for the Arts and Humanities (DARIAH, 2.4 million euro per year). CLARIN aims to provide easy and sustainable access for scholars in the humanities and social sciences to digital language data (in written, spoken, video or multimodal form) and advanced tools to discover, explore, exploit, annotate, analyse or combine them, independently of where they are located. To this end CLARIN is in the process of building a networked federation of European data repositories, service centres and centres of expertise, with single sign on access for all members of the academic community in all participating countries. DARIAH aims to facilitate long-term access to, and use of, all European arts and humanities digital research data. The DARIAH infrastructure will be a connected network of people, information, tools and methodologies for investigating, exploring and 
supporting work across the broad spectrum of the digital humanities. Both of these infrastructures are still being built, with a growing number of Member States.

While these developments must be considered a success, the humanities are still struggling for their place in the Framework Programmes. The Seventh Framework Programme 2007-13 (FP7) did call on the humanities for policy advice, but the calls were limited and funding was a fraction of that available for science and technology. Nevertheless FP7 marked a significant recognition of the humanities as part of the knowledge base for policy makers, which has opened up the battle for budgets and action lines in research programmes.

A number of representative bodies and reports have sought to outline how the humanities may turn programmatic intentions of social relevance into reality. In 2007 the Standing Committee for the Humanities at the ESF (European Science Foundation) published a position paper on the nature and importance of the humanities, pointing out the prominent role played by research on 'communicative systems':

The humanities focus on 'the human element' in the physical, biological, mental, social and cultural aspects of life. They attempt to provide insights into how knowledge arises from the constant interaction between individual and society. When studying culture, the humanities engage not just with its present manifestations, but also with those of the past. All culture comes to us from the past. If traditions, memories and ongoing practices are supplemented and reshaped by individual choices, those in turn are constrained by structural features of the various cognitive and value systems we employ. In this respect we are the product of our past, of the structural properties of our present environment, of our characteristically human capacities such as language, perceptual and communicative systems, and of our bodies.

The document further stressed the significance of the past for contemporary (and future) culture and pointed out the prominent role played by research on 'communicative systems' in physical, biological, mental, social and cultural aspects of life. ${ }^{31}$

Recently, the ESF has been largely replaced by Science Europe as the main association of European funding organisations, and its Committee for the Humanities has developed a focus on the cognitive role of the humanities in society. In its very first statement on societal challenges the Committee singles out 'understanding and influencing behavioural 
change' as a broad research theme which should be imbedded in all research areas, such as energy consumption, food, health and transport. The Committee also sees a direct economic value of the humanities in understanding and developing innovation ecosystems:

Technologies are shaped by human involvement and in many cases the human aspects of innovation development and uptake are as challenging as the technological aspects. Design is a crucial aspect in the development of products and services, which is under-researched. This theme would include work on how innovation occurs in different areas of work, why some innovations are successful while others fail, and why some societies are more innovative than others - in short, how do we 'make' innovation?

The Committee further argues that humanities research in innovation will bring out the wider social, political and cultural contexts, 'recognising that the value of innovation should not be measured purely in economic terms' ${ }^{32}$ However, it seems fair to say that, while the 2007 ESF document fitted comfortably with statements by our interviewees of the broad social and cultural value of the humanities, the 2013 Science Europe document is a clear statement of direct economic, social and cultural usefulness. Perhaps it is an indication that with increasing engagement in political processes utilitarian arguments become more important.

Meanwhile, the EU process continued to be informed by internal reports. An expert group on the humanities reported in 2007 on how the humanities might contribute to already defined tasks in FP7 and recommended with some success that the wording in the calls be made 'more humanities friendly'. ${ }^{33}$ In 2009 the METRIS report for the Commission highlighted that a lack of data 'is the first impediment to a proper understanding of the evolving role of SSH in society'. ${ }^{34}$ An evaluation of SSH research in FP5 and FP6, published in 2010, concluded that EU funding is instrumental in creating a European Research Area both in terms of significantly increasing funding levels and fostering cross-national collaboration and mobility. The report also highlighted the benefits of engaging policy makers with ongoing research. However, policy makers found that researchers in social sciences in their countries collaborate more than researchers in the humanities. Research in the humanities was perceived as having a national focus, and collaboration with researchers abroad is, if at all, only carried out with those in neighbouring countries.' Policy makers reported that there is little 
formal engagement between researchers and policy makers. In general, the need for research stated by most national authorities

... seems to be on information and data on current issues which are often high on the agenda of those Ministries dealing with public finances. For example, gauging the state of the economy or understanding the issues affecting employment. Many policy makers interviewed acknowledged that there was potential and good rationale for using SSH research in this context. More specifically, policy makers in the Eastern European Member States explained the need for SSH research to assess the impacts on changes in society and lifestyles of citizens. Specific examples of this included examining the options and choices for the development of a knowledge-based society and the implications of European integration and enlargement for governance and citizens. ${ }^{35}$

In 2011 the Commissioner for Research and Innovation announced that the next Framework Programme, Horizon 2020, to be launched in 2014, would not have a specific grand challenge for the SSH and that instead they would be 'mainstreamed across research challenges of climate change, energy, food, health, security and transport'. Broad segments of the SSH community saw the announcement as a threat that SSH research would only become a fig leaf or an add-on to science- and technologydriven research. For the first time a broad coalition of SSH organisations joined forces in a European Alliance for the Social Sciences and Humanities, EASSH, and drafted an open letter to the Commissioner. More than 25,000 SSH researchers signed the letter, arguing that:

While for many questions, natural, human and social sciences need to join forces, there are also important societal and economic transformations, which can be described as Social Sciences and Humanities (SSH)-centred challenges: they regard areas as diverse as education, gender, identity, intercultural dialogue, media, security, social innovation, to name but a few. Similarly, only SSH research can address many of the key behavioural changes and cultural developments which provide the backdrop to the EU's current approach to 'Tackling Grand Societal Challenges', such as for example changing mindsets and lifestyles, models for resilient and adaptive institutions, or the evolving position of Europe in a global context. ${ }^{36}$

The Commission did change tack and promised to include a sixth challenge. In May 2012 the Council of Ministers for Research decided to 
include, as a societal challenge, 'Europe in a changing world: Inclusive, innovative and reflective societies', which 'will support social sciences and humanities research'. In addition, the Ministers confirmed that the humanities and social sciences should be mainstreamed across all the grand challenges of Horizon 2020. ${ }^{37}$ At the time this seemed like a significant victory for the SSH action but subsequent developments showed that the European decision-making process is often opaque. The positive wording in Horizon 2020 on the value of the humanities and social sciences may be little more than window- dressing. It is clear that the promise of 'mainstreaming' does not ensure anything but ornamental additions to science projects. When the work programme for the sixth challenge, 'Europe in a changing world: inclusive, innovative and reflective societies', was launched in December 2013 it was clear that the budget would be quite small, in the region of 400 million euro, while other challenges would be allocated several billion euro each. However, the Commissioner was very clear in her call on the humanities:

Europe is still facing many long-term and complex challenges. It takes profound knowledge and insight to really understand these challenges and how they affect us, and to guide us to solutions. That is why the social sciences and humanities are more essential than ever, and why we, as policy makers, are keen to have their contribution. We need them to understand ourselves, our society and the challenges we face. We need them to guide politicians and policy makers and to inform public opinion. Research and technology provide many answers to the challenges we face, but technological fixes alone aren't enough to solve our major, complex problems. A knowledge society needs to know itself, and the social sciences and humanities are the keys to this. ${ }^{38}$

On a global scale the European Union is a unique expression of a political system calling on the SSH for policy advice while also allocating substantial funds for bottom-up research funding. In the last 10-15 years humanists in Europe have engaged in political processes to argue the societal importance of their research and they have had some success in developing an agenda and a legitimate role for the humanities at negotiating tables. It is notable that EU programmes will now call on the humanities to contribute insights into major societal challenges such as health, climate, food and transport. On the other hand, it is clear that, despite some political goodwill, there is considerable resistance or lack of appreciation at many political and bureaucratic levels. The actual wording of work programmes and calls for funding is a battle ground 
that is still very often held by technocrats who have little appreciation of humanities research. While the humanities now have several important organisational voices, the European process requires follow-up and lobbying, which is still beyond the capacity of the humanities.

\section{Conclusion: the politics of the humanities}

The humanities have a unique position in global politics. On the one hand, many politicians increasingly recognise that at the heart of all the grand challenges of the 21st century are questions of human motivation, behaviour and choice. On the other hand, the academic disciplines that wholeheartedly focus on the human are rarely, if ever, called on to inform the political system. Evidently, in many corners of the world the humanities are exposed to certain societal expectations, even if they are not as great many humanists would wish and, in some places, they are far too low. It is also obvious that the humanities deal with themes of high demand, such as cultural identity and heritage, and that this is also what many humanist scholars think they should be dealing with. To be sure, there is still a mismatch between supply and demand. Yet, it appears to be more a matter of quantity (i.e. scarcity of funding) than of quality (i.e. topics addressed).

The humanities cannot expect this situation to change without action. In the EU the humanities have benefited from a close alliance with the social sciences and from a sustained lobbying process, which has included a large number of actors. On a global scale, the EU's recognition of the humanities is unique, expressed in terms of financial support for basic and targeted research and infrastructure, and the words of support from the political side provides a strong contrast to its neglect in the United States and many other countries. In countries like South Africa and Australia, on the other hand, political attention to the humanities has not been without a cost, and the new embrace of the humanities by the Chinese authorities potentially raises ethical problems and questions of freedom of research that may carry a global lesson.

Critics of the rapprochement of the humanities to grand challenge social, political and cultural issues observe that the humanities may lose their way by becoming too utilitarian or embroiled in political expediency. Indeed, the differences between the European and American humanities seem to us to be widening as a result of this process. The American focus on traditional humanities' virtues, such as individualism and book publication, is clearly at odds with a European culture that is increasingly project-funded, goal-oriented and aimed at peer-reviewed 
journal publication. Critics may see the European way as a Faustian deal. The critique that has been voiced against current research policy in Europe and elsewhere most often concerns the deliberate commodification and commercialisation of scientific research - though, paradoxically, this policy is not pursued in the spirit of the free market, but goes hand in hand with the audit society, with a strong emphasis on planning and control. ${ }^{39}$ One of the harmful outcomes of this policy is a transformation of scientific researchers into experts, better suited for an R\&D department in a big company than for academia. ${ }^{40}$ In order to serve human interests in the broad sense, professionals 'should not have values regarding the development of society; they should not be political', as stated by one critic of research policy. ${ }^{41}$ One way of avoiding value bias in research is to keep a certain distance between researchers and those who expect scientific results to be in line with their ideologies or interests. This means that the research community should not get too involved with stakeholders outside academia; nor should it let them interfere with the scientific choice of themes and methods to be applied. Such at least is the view of some critics of current research policy trends in many countries. ${ }^{42}$

On the other hand, a more positive view would see a culture change as a way to revitalise the humanities. In the autumn of 2013, Helga Nowotny, President of the European Research Council, commented on the contrast between the EU and the US in political attitude to the SSH:

Under its new EU research programme, Horizon 2020, the importance of the social sciences and humanities has been formally recognized... More than $€ 28 \mathrm{bn}$ is being allocated to tackle societal challenges, including energy efficiency, climate change, health, ageing, security, privacy issues and digitization.... It is obvious that the social science and humanities have a lot to contribute to each of these agendas, and the EU's integrative approach is laudable... the Horizon 2020 programme reflects a strikingly different approach to developments across the Atlantic. In the United States, the social sciences and humanities are under attack. In Europe we are committed to integrating the natural sciences, engineering, and social sciences and humanities... ${ }^{43}$

Similarly positive is the 'Vilnius declaration', conveyed by a consortium of European humanists and social scientists at an EU conference in Vilnius in September 2013. The declaration stresses the importance, significance and even the indispensability of the humanities for 
addressing profound current societal challenges and applauds the mainstreaming of the humanities across all six priorities of Horizon 2020:

European Social Sciences and Humanities are world class, especially considering their diversity. They are indispensable in generating knowledge about the dynamic changes in human values, identities and citizenship that transform our societies. They are engaged in research, design and transfer of practical solutions for a better and sustainable functioning of democracy. Their integration into Horizon 2020 offers a unique opportunity to broaden our understanding of innovation, realigning science with ongoing changes in the ways in which society operates.

Furthermore, the Vilnius declaration supports assessment of impact as one of the basic quality criteria of good science, although it is not made perfectly clear whether such assessment is to be one of the criteria for the allocation of research resources, or if it is a post festum measure to find out if research results are disseminated and implemented. ${ }^{44}$

So, looking at policy developments in the EU and comparing them to the US, we might find some cause for optimism, even a model for other regions to follow. But, as we saw above, these same developments have their critics. As the authors of this report, where do we stand? The more positive view - in favour of greater engagement between humanists and policy makers - fits well with some of our findings in Chapter 2 , where over half of our interviewees identified 'social value' as the most important extrinsic justification for the humanities, defined as a broad concept ranging from moral values to informing social decisionmaking and contributing to or contesting social cohesion. Another very frequently stressed value was 'cultural heritage', implying the preservation and critical evaluation of material as well as immaterial leftovers of the past. We also saw in Chapter 3 that much humanities research genuinely engages with the social in terms of the themes selected for study.

But there are at least two problems with which we should close. First, in this chapter we have also seen that some policy makers take a much narrower view of the contribution (if any) that the humanities have to make, particularly in promoting economic growth and innovation. The problem is that, as we saw in cChapter 2, our interviewees rarely identified such values and showed very little appetite for seeing them as a goal for humanities research. So, whenever the more narrow-minded policy makers have the upper hand, there will be a serious rift with the humanists. Second, even when policy makers take the broader view and stress 
the sorts of social goals discussed above, there is a danger that humanists will end up making a Faustian pact; so, how far should they allow their research to be guided by the goals and interests of policy makers?

Clearly, humanities politics are not just a question of raising the voice in favour of more funding. There are issues of social and political engagement that to many humanists may seem alien to what they signed up for and alien to academic life. However, the question will not go away and the discussion is vital to both the future of the humanities and how society will benefit from the humanities.

(c) (i) Except where otherwise noted, this work is licensed under a a copy of this license, visit http://creativecommons.org/licenses/by/3.0/ 